\title{
Type-dependent action modes of TtAA9E and TaAA9A acting on cellulose and differently pretreated lignocellulosic substrates
}

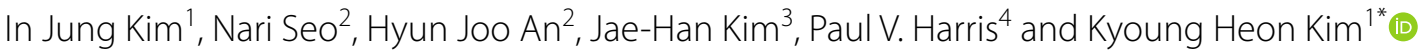

\begin{abstract}
Background: Lytic polysaccharide monooxygenase (LPMO) is a group of recently identified proteins that catalyze oxidative cleavage of the glycosidic linkages of cellulose and other polysaccharides. By utilizing the oxidative mode of action, LPMOs are able to enhance the efficiency of cellulase in the hydrolysis of cellulose. Particularly, auxiliary activity family 9 (AA9) is a group of fungal LPMOs that show a type-dependent regioselectivity on cellulose in which Types 1, 2, and 3 hydroxylate at C1, C4, and C1 and C4 positions, respectively. In this study, we investigated comparative characteristics of TtAA9E from Thielavia terrestris belonging to Type 1 and TaAA9A from Thermoascus aurantiacus belonging to Type 3 on cellulose and pretreated lignocellulose.

Results: From product analysis, TtAA9E dominantly generated oligosaccharides with an aldonic acid form, which is an evidence of $\mathrm{C} 1$ oxidation, while TaAA9A generated oligosaccharides with both aldonic acid and 4-ketoaldose forms, which is evidence of $\mathrm{C} 1$ and $\mathrm{C} 4$ oxidations, respectively. For hydrolysis of cellulose (Avicel) by cellulase, higher synergism was observed for TtAA9E than for TaAA9A. For hydrolysis of pretreated lignocellulose using rice straw, synergistic behaviors of TtAA9E and TaAA9A were different depending on the pretreatment of rice straw. Specifically, on acid-pretreated rice straw, TtAA9E showed a higher synergism than TaAA9A while on alkali-pretreated rice straw, TaAA9A showed a higher synergism than TtAA9E.

Conclusions: We show type-dependent action modes of TtAA9E and TaAA9A for cellulose oxidation together with substrate-dependent synergistic hydrolysis of cellulosic substrates. The results obtained from this study indicate the different behaviors of AA9s on cellulose and pretreated lignocellulose, suggesting a selection of AA9 proteins specific to substrates is required for industrial utilization.
\end{abstract}

Keywords: Auxiliary activity family 9, Lytic polysaccharide monooxygenase, Glycoside hydrolase family 61, Cellulose oxidation, Synergism, Cellulose hydrolysis

\section{Background}

Recently, the fortification of cellulase preparations by adding synergistic proteins was shown to be effective in facilitating the enzymatic saccharification of lignocellulose [1]. Synergistic proteins lack hydrolytic ability but

\footnotetext{
*Correspondence: khekim@korea.ac.kr

1 Department of Biotechnology, Korea University, Graduate School, Seoul 02841, South Korea

Full list of author information is available at the end of the article
}

play a significant role in promoting cellulase activity in the hydrolysis of lignocellulose [1-3]. Some of the synergistic proteins were reported to increase the accessibility of cellulose to cellulase through the modification or disruption of crystalline regions which are typically less inaccessible to cellulase [4]. Utilization of highly efficient synergistic proteins can reduce the total cellulase loadings required to achieve an industrially applicable cellulose conversion (i.e., 80-90\%), thus reducing the cost for saccharification of lignocellulose [3]. 
There has been much attention on the lytic polysaccharide monooxygenases (LPMOs), a family of recently discovered synergistic proteins. The distinguishing feature of LPMOs is their unique oxidative cleavage action on polysaccharides [5-7]. Cellulose cleavage by LPMOs is known to involve the reduction of $\mathrm{Cu}^{2+}$ at the active site and the abstraction of $\mathrm{H}$ and hydroxylation $[5,6,8]$. Due to their oxidative mode of action, LPMOs require external electron donors such as ascorbic acid, reduced glutathione, gallate, or sodium azide for their activity $[6,7$, 9]. In addition to these synthetic reducing agents, lignin, which naturally exists in lignocellulose, or enzymes such as cellobiose dehydrogenase $(\mathrm{CDH})$ and glucose-methanol-choline oxidase/dehydrogenase (GMC) family of oxidoreductase can also act as reducing agents for LPMOs $[8,10-13]$. LPMOs target the crystalline region of the cellulose surface which is typically more recalcitrant to cellulase action. This surface oxidation by LPMOs may induce structural modification of the recalcitrant cellulose, making it more amendable to subsequent hydrolysis by cellulase through the creation of additional chain ends $[14,15]$.

Family auxiliary activity 9 (AA9), previously known as glycoside hydrolase family 61 (GH61), is the fungal family of LPMOs active on cellulose [3, 7, 9, 16, 17]. Currently, AA9s are supplemented to some commercial cellulase preparations, where they have been shown to synergise with cellulase at industrially applicable levels of cellulose hydrolysis yields (i.e., 80-90\%) [3]. Based on sequence similarity, AA9s are categorized into Types 1, 2, and 3, and each type recognizes different sites of cellulose for oxidation. Specifically, Types 1 and 2 predominantly utilize $\mathrm{C} 1$ (reducing end) and C4 (non-reducing end) oxidations, generating oxidized oligosaccharides in the form of aldonic acid and 4-ketoaldose, respectively [5, 8, 18, 19]. Type 3 catalyzes both $\mathrm{C} 1$ \& $\mathrm{C} 4$ oxidations, generating both aldonic acid and 4-ketoaldose forms of cellooligosaccharides [18].

The molecular functions of Type 1 TtAA9E from Thielavia terrestris and Type 3 TaAA9A from Thermoascus aurantiacus were characterized earlier [3, 6, 20]. Although the direct oxidative action of TaAA9A was already studied through product analysis, that of TtAA9E has not been studied [6]. The structure of both AA9s was determined, and both were shown to improve the hydrolytic efficiency of lignocellulosic biomass by cellulase [3, 6]. Especially, the expression of TaAA9A along with cellulase in a commercial cellulolytic strain of Trichoderma reesei enabled the reduction of cellulase loadings to a half of that required by a strain expressing only cellulase [3]. Furthermore, their oxidative actions were verified by the increase of synergistic activity of those AA9s in the presence of reducing cofactors such as gallate, lignin, ascorbic acid, or CDH in the hydrolysis of cellulose and pretreated lignocellulose [6, 11, 20]. With their high synergistic activity, TtAA9E and TaAA9A are industrially applicable.

In order to better understand the oxidative and synergistic activities of TtAA9E and TaAA9A, in this study, we have comparatively examined these two AA9s. First, reaction products which were directly generated from cellulose with AA9s were analyzed using matrix-assisted laser desorption/ionization-tandem time-of-flight mass spectrometry (MALDI-TOF/TOF MS) to investigate the oxidative mode of cleavage actions of TtAA9E and TaAA9A. Second, their synergistic activities with cellulase were studied against pure cellulose (Avicel) and rice straw samples using two different pretreatments (e.g., acid- and alkali-pretreatments as the two representative pretreatment methods). It is because pretreated lignocellulose varies in its composition and physical properties that could significantly affect the synergistic hydrolysis of AA9s [10-12]. Our comparative study of these two different AA9s in the oxidation of cellulose and the synergism with cellulase will shed light on the substrate specificities and functional variations of AA9s, which in turn will be helpful for the customization of AA9s in their industrial applications.

\section{Results and discussion \\ Oxidative cleavage of cellulose by TtAA9E and TaAA9A}

To investigate the direct enzymatic activities of TtAA9E and TaAA9A towards cellulose, the reaction products from pure cellulose were analyzed by MALDI-TOF/TOF MS (Fig. 1). Our studies using Avicel revealed that both TtAA9E and TaAA9A cleave crystalline cellulose in the presence of $10 \mathrm{mM}$ ascorbic acid to produce oxidized oligosaccharides with various degrees of polymerization (DPs). However, the two AA9s produce distinctively different product profiles (Fig. 1). These results indicate that both AA9s have the endo-type oxidative cleavage mode but at the same time different oxidative regioselectivities toward $\beta$-glycosidic linkages on cellulose.

To verify their oxidative regioselectivities, oxidized sugars were identified by mass-based prediction of the possible products based on a previous report (inset in Figs. 1, 2) [19]. Possible products of $\mathrm{C} 1$ oxidation by LPMOs can be aldonic acid ( $M+16$, where $M$ indicates the mass of native oligosaccharides), sodium adduct of aldonic acid $(\mathrm{M}+16-\mathrm{H}+\mathrm{Na})$, and 1,5 $\delta$-lactone $(\mathrm{M}-2)$ whereas 4-ketoaldose $(\mathrm{M}-2)$ and gemdiol $(\mathrm{M}+16)$ are generated by $\mathrm{C} 4$ oxidation. In the case of $\mathrm{C} 1 \& \mathrm{C} 4$ oxidations, all of the above-listed sugars and doubly oxidized sugars containing both aldonic acid and gemdiol $(\mathrm{M}+32), 1,5 \delta$-lactone and gemdiol $(\mathrm{M}+14)$, or aldonic acid and 4-ketoaldose $(\mathrm{M}+14)$ can be observed (Fig. 2) [19]. In this study, TtAA9E, whose products have not 

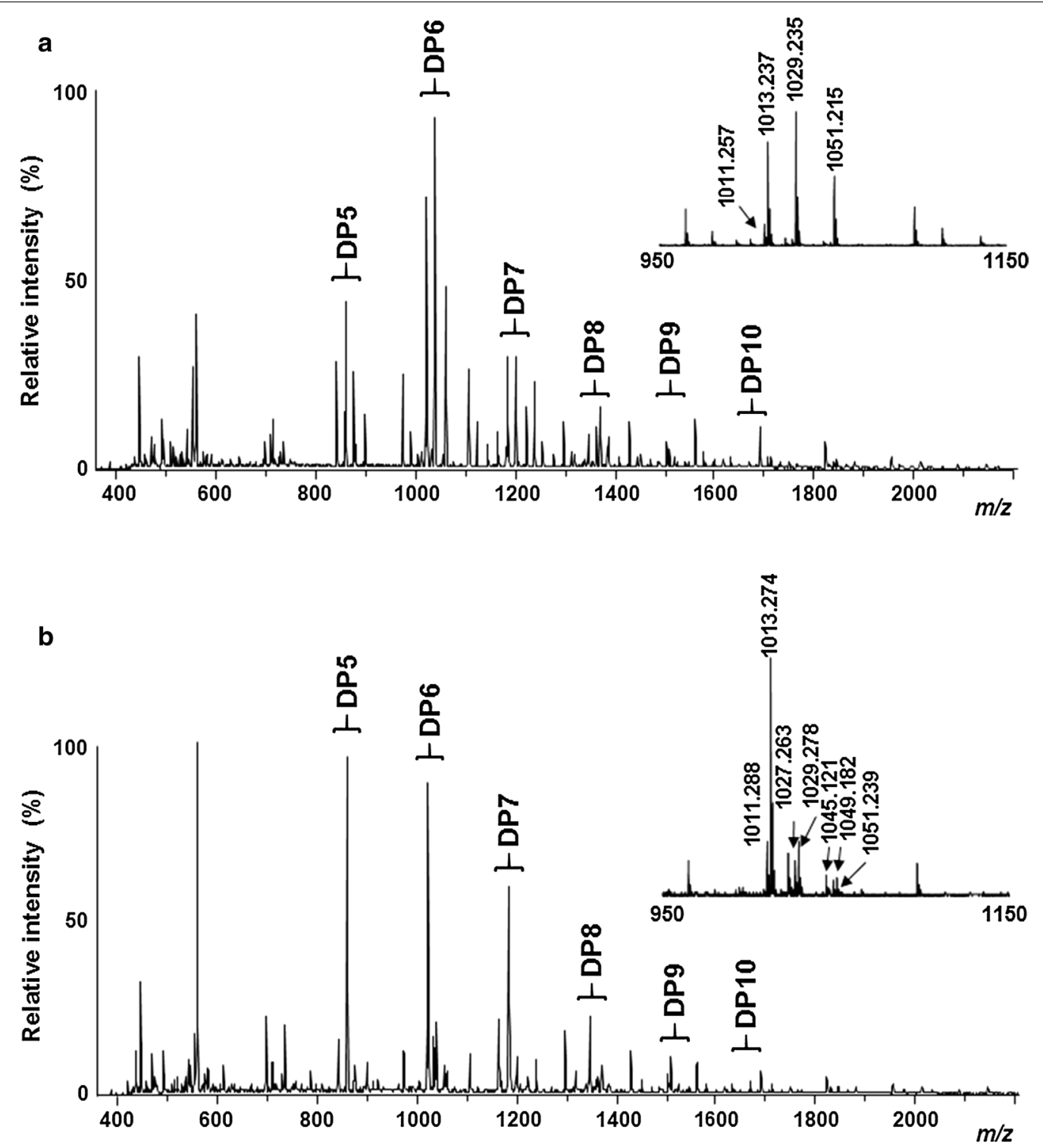

Fig. 1 Product analysis obtained from the incubation of cellulose with TtAA9E or TaAA9A using MALDI-TOF/TOF MS. Avicel (5\%, w/v) was incubated with TtAA9E or TaAA9A (1 mg/g Avicel) in $50 \mathrm{mM}$ sodium acetate ( $\mathrm{pH}$ 5.0) with $10 \mathrm{mM}$ of ascorbic acid at $50{ }^{\circ} \mathrm{C}$ for 4 days. Overall profiles of products with sodium saturation generated from the incubation of cellulose with $\mathbf{a}$ TtAA9E or $\mathbf{b}$ TaAA9A in the presence of $10 \mathrm{mM}$ ascorbic acid. Insets are expanded mass spectra for DP6 products obtained from the incubation of cellulose with a TtAA9E or $\mathbf{b}$ TaAA9A. 100\% relative intensity represents $3.31 \times 10^{4}$ and $2.62 \times 10^{4}$ arbitrary units (a.u.) for the full spectra of TtAA9E and TaAA9A, respectively, and $3.31 \times 10^{4}$ and $2.24 \times 10^{4}$ a.u. for the close-up peaks of DP6 for TtAA9E and TaAA9A, respectively

been reported in the literature, dominantly produced aldonic acid forms of oligosaccharides (Fig. 1a). The peak corresponding to $\mathrm{M}-2$ is likely to be the unstable lactone form of aldonic acid rather than 4-ketoaldose, considering the exclusive formation of aldonic acid and its sodium adduct by the action of TtAA9E. In addition, double oxidized sugar was not observed with TtAA9E, which argues against $\mathrm{C} 1$ and $\mathrm{C} 4$ oxidations. These results indicate the strict $\mathrm{C} 1$ oxidative mode of TtAA9E. In this study, TaAA9A showed a very low cleaving activity on Avicel, and its full spectrum was difficult to distinguish from that of the control, which was incubated without TaAA9A (data not shown). Nevertheless, the masses corresponding to possible non-reducing end-oxidized and doubly oxidized sugars $(\mathrm{M}-2, \mathrm{M}+14$, and $\mathrm{M}+32)$ and aldonic acid forms were obtained with TaAA9A, which were not observed with the control (Fig. 1b; Additional file 1: Figure S1). 


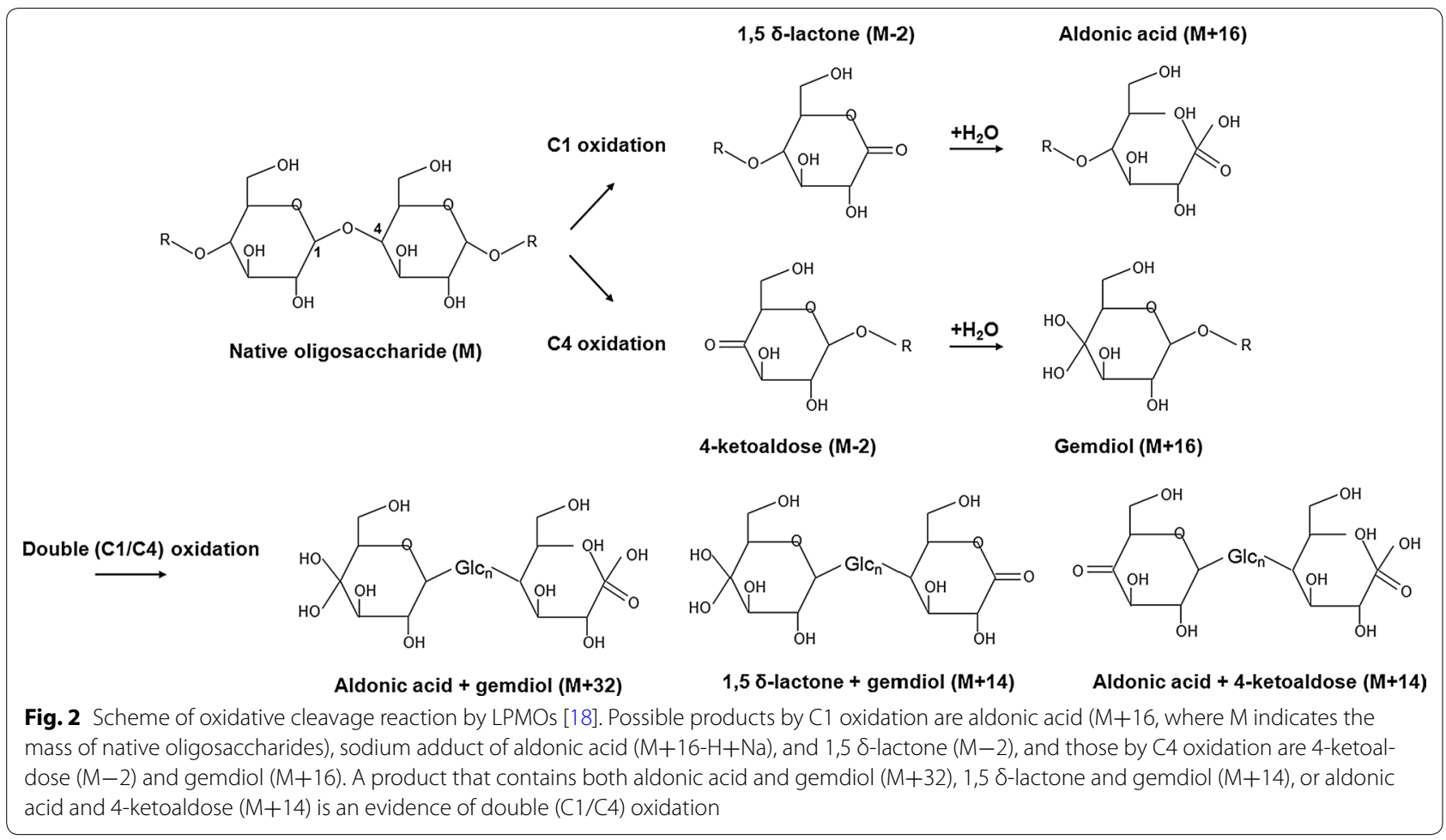

Although the peak corresponding to M-2 could indicate either reducing end oxidation $(1,5 \delta$-lactone of aldonic acid) or non-reducing end oxidation (4-ketoaldose), doubly oxidized sugar $(M+14$ and $M+32)$ ensures the occurrence of oxidations both at reducing and nonreducing ends by $\mathrm{TaAA} 9 \mathrm{~A}$, something not observed with the strict C1-oxidizing TtAA9E. This is consistent with the previous study with TaAA9A, in which TaAA9A was shown to oxidize at both reducing and nonreducing ends of phosphoric acid-swollen cellulose (PASC) [6]. Thus, TaAA9A was considered to use the $\mathrm{C} 1$ and $\mathrm{C} 4$ oxidation mode in this study.

AA9s are known to possess a type-dependent selective mode of action for oxidation of cellulose: Type 1 dominantly oxidizes $\mathrm{C} 1$, Type 2 dominantly oxidizes $\mathrm{C} 4$, and Type 3 can oxidize both $\mathrm{C} 1$ and $\mathrm{C} 4$ [18]. This close correlation between the sequence/structure-based classification and the oxidation mode agrees with our results from the product analysis of TtAA9E and TaAA9A. Specifically, TtAA9E belonging to Type 1 induces an oxidative cleavage of $\beta$-1,4-glycosidic bonds of cellulose via $\mathrm{C} 1$ oxidation while TaAA9A belonging to Type 3 cleaves the $\beta-1,4$-glycosidic bonds via both $C 1$ and $C 4$ oxidations. The generation of oxidized products by TtAA9E was much higher compared to that by TaAA9A (Additional file 1: Figure S1). Through the cleavage action of TtAA9E, TtAA9E was shown to have a higher PMO activity.

\section{Synergistic activity of TtAA9E and TaAA9A on the enzymatic hydrolysis of cellulose}

From the product analysis earlier in this study, different modes of action were observed for the two AA9s when they were directly applied to cellulose (Fig. 1). In this study, furthermore, the synergistic effects of TtAA9E and TaAA9A on the enzymatic hydrolysis of pure cellulose were investigated (Fig. 3). Avicel was hydrolyzed by a commercial cellulase preparation, Celluclast $1.5 \mathrm{~L}$ either with or without AA9s in the presence of antibiotic $\mathrm{NaN}_{3}$. In contrast to a previous study of TtAA9E and TaAA9A [3], which reported no synergistic activity of these two AA9s on pure cellulose without adding reducing agents, the two enzymes showed synergism with cellulase in this study. This is possibly because the antibiotic $\mathrm{NaN}_{3}$ in the reaction mixture was also able to work as the reducing power for AA9s activities as described in an earlier study [9]. The synergistic activity on cellulose was higher for TtAA9E than for TaAA9A. At a cellulase loading of $0.9 \mathrm{FPU} / \mathrm{g}$ Avicel, the reducing sugar yields with TtAA9E and TaAA9A were 1.9 and 1.2 fold higher than the control without AA9s in the hydrolysis, respectively $(p<0.05)$. The higher synergistic activity of TtAA9E was consistent with its higher cleavage activity observed in the product analysis earlier in this study.

Even with the experimentally determined relation between AA9 activity on cellulose and the synergism with cellulase in this study, it is difficult to generalize how type-dependent regioselectivity is associated with 


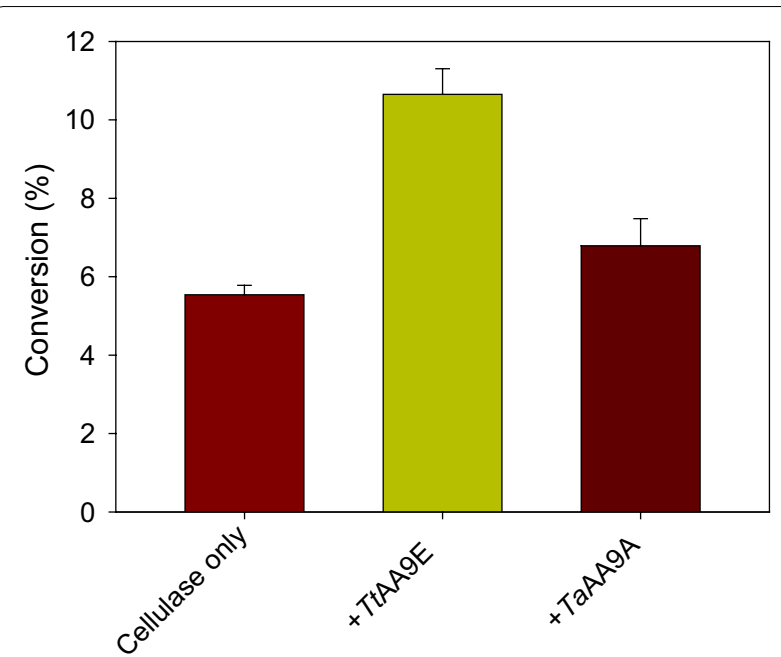

Fig. 3 Synergistic activities of TAAA9E and TaAA9A in the hydrolysis of cellulose by cellulase. Avicel (1\%, w/v) was hydrolyzed using Celluclast $1.5 \mathrm{~L}$ (0.9 FPU/g Avicel) without (control), with TtAA9E, or with TaAA9A (1 mg/g Avicel) in 50 mM sodium acetate buffer ( $\mathrm{pH}$ 5.0) containing $\mathrm{NaN}_{3}$ at $50^{\circ} \mathrm{C}$ for $48 \mathrm{~h}$. The reducing sugar was determined by DNS assay, and the reducing sugar yields were expressed as \% of the theoretical maximal yield of glucose. The data represent means \pm standard errors obtained from the three experimental replicates

the synergistic activity of AA9s with cellulase. Only a few AA9s such as TtAA9E, TaAA9A, PcGH61D, StCel61a, and $C g A A 9$ have been studied for their synergistic activities $[3,9,20,21]$. In addition, those studies on synergism were performed under various enzymatic reaction conditions such as different loadings of cellulase and AA9, different types and concentrations of reducing cofactors and cellulosic substrates and so on, which might have highly affected synergistic characteristics [1].

\section{Synergistic activity of TtAA9E and TaAA9A on the enzymatic hydrolysis of pretreated lignocellulose}

Depending on the lignocellulose pretreatment method used, pretreated lignocellulosic substrates have significantly different compositions and physical properties as a result of the pretreatment. The resulting composition and physical properties affect the mode of action of enzymatic hydrolysis of the pretreated lignocellulose, by which enzymes target and hydrolyze the pretreated lignocellulose [22-24]. Indeed, AA9s have been reported to display different behaviors against different pretreated lignocellulosic substrates [10-12]. Therefore, following the synergistic study on cellulose, comparison was made for the synergism of TtAA9E and TaAA9A in the enzymatic hydrolysis of pretreated lignocellulose (Fig. 4) although these two AA9s had been already assessed for their synergistic activities on other pretreated lignocellulose such as acid-pretreated corn stover or steam-exploded birch before $[3,25]$. Two types of rice straw pretreated using dilute sulfuric acid (ACID) and aqueous ammonia (ALKALI), which had different compositions mainly in terms of cellulose, hemicellulose, and lignin, were generated in this study [26]. As described in Methods, ACID had a lower content of hemicellulose, but the lignin content in ACID was higher than that in the untreated rice straw. In contrast, ALKALI had a lower content of lignin than ACID.

Both TtAA9E and TaAA9A showed a synergistic activity in the enzymatic hydrolysis of ACID and ALKALI in

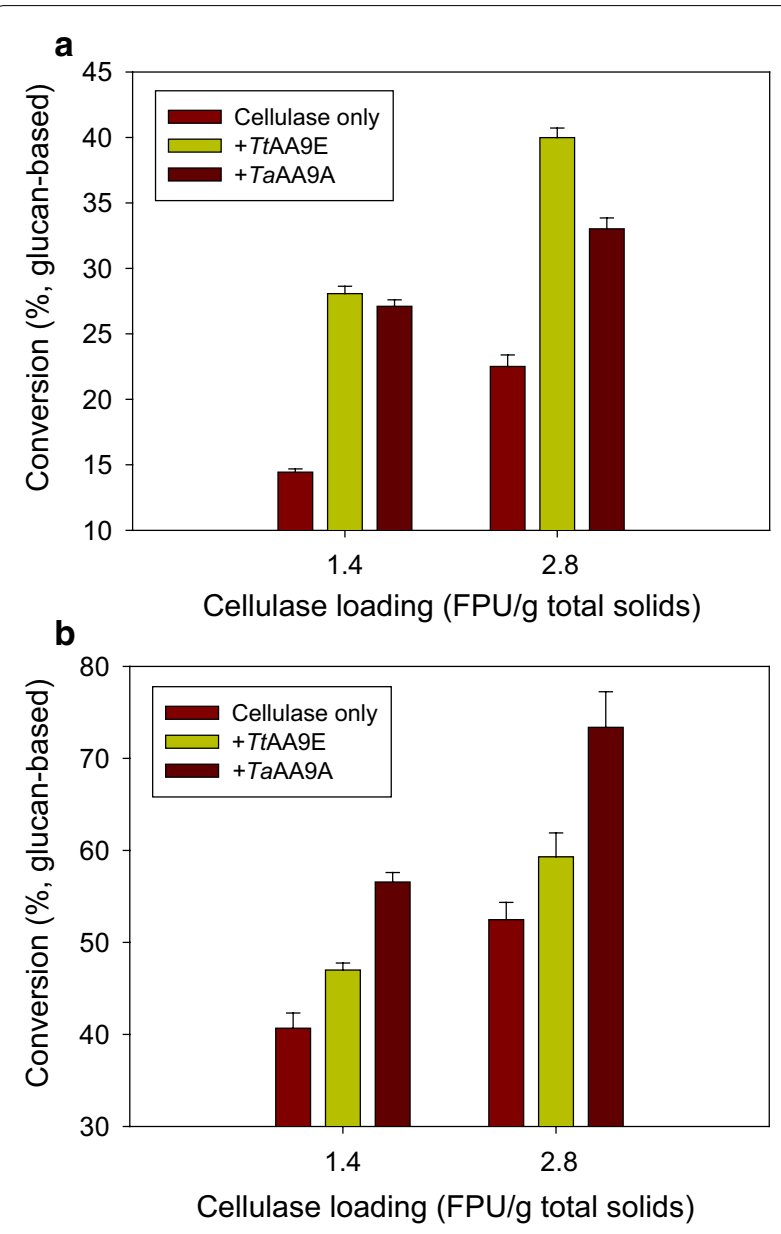

Fig. 4 Synergistic activity of TtAA9E or TaAA9A in the hydrolysis of two types of pretreated rice straw by cellulase. A solid loading of $5 \%$ $(\mathrm{w} / \mathrm{v})$ of pretreated rice straw of a ACID or b ALKALI was hydrolyzed using Celluclast $1.5 \mathrm{~L}$ (1.4 and $2.8 \mathrm{FPU} / \mathrm{g}$ total solids) in the absence or presence of TtAA9E or TaAA9A ( $5 \mathrm{mg} / \mathrm{g}$ cellulose) in $50 \mathrm{mM}$ sodium acetate buffer ( $\mathrm{pH}$ 5.0) in the presence of $0.02 \%$ of $\mathrm{NaN}_{3}$ and $1 \mathrm{mM}$ of $\mathrm{MnSO}_{4}$ at $50{ }^{\circ} \mathrm{C}$ for 8 days. The reducing sugar was determined by DNS assay, and the reducing sugar yields were expressed as $\%$ of the theoretical maximal yield of glucose. The data represent means \pm standard errors obtained from the three experimental replicates 
the presence of cellulase at 1.4 and $2.8 \mathrm{FPU} / \mathrm{g}$ total solids (Fig. 4). As for TaAA9A, regardless of its very low cleavage activity as shown in Fig. 1, its synergistic effect was exhibited with the pretreated rice straw. This could be because TaAA9A might have induced the surface disruption of the pretreated rice straw substrates despite of the fact that TaAA9A produced almost undetectable amounts of sugar products [4]. This disruption of the pretreated rice straw substrates by TaAA9A could have been led to the facilitation of the hydrolysis of pretreated rice straws by cellulase.

TtAA9E (1.9 or 1.8 fold) showed a higher synergistic activity than TaAA9A (1.9 or 1.5 fold) on ACID, whereas the opposite results were obtained on ALKALI (1.1 or 1.2 fold in case of TtAA9E and 1.4 fold in case of TaAA9A) with cellulase loading of 1.4 or $2.8 \mathrm{FPU} / \mathrm{g}$ total solids, respectively. These results indicate that the synergistic activity of AA9s is specific for pretreatment types and is highly affected by the nature of the pretreated lignocellulose, which is often represented by its composition and physicochemical properties [10-12]. Therefore, to obtain higher hydrolysis yields, the employment of AA9s needs to be customized for the types of pretreatment and lignocellulose.

\section{Conclusions}

In this study, we have performed a comparative study on the cellulose oxidative and synergistic activities of TtAA9E and TaAA9A, which are phylogenically Types 1 and 3 AA9s, respectively. The characterization of TtAA9E and TaAA9A on cellulose revealed that their regioselectivity on $\beta-1,4$-glycosidic bonds depends on their type. Aldonic acid derived from $\mathrm{C} 1$ oxidation was produced by the Type 1 AA9 (in case of TtAA9E), and aldonic acid and 4-ketoaldose derived from $\mathrm{C} 1$ and $\mathrm{C} 4$ oxidations were produced by the Type 3 AA9 (in the case of TaAA9A). In addition, these oxidative cleaving activities on cellulose were higher in TtAA9E than in TaAA9A. TtAA9E and TaAA9A exhibited different behaviors with regard to their synergistic activity with cellulase in the hydrolysis of both cellulose and pretreated lignocellulose. TtAA9E showed a higher synergistic activity on cellulose, which was consistent with its higher cleaving activity observed from the product analysis of direct actions of the AA9s on cellulose. In summary, different functional properties of the two distinctive AA9s were demonstrated using cellulose and pretreated lignocellulose as substrates. Our results suggest that the systematic customization of AA9s depending on the pretreatment types and biomass is necessary to maximize the hydrolysis efficiency and performance of AA9s.

\section{Methods}

\section{AA9s and cellulase and pretreated rice straw}

TtAA9E and TaAA9A used in this study were provided by Novozymes (Bagsvaerd, Denmark). For the assay of synergistic activity in the enzymatic hydrolysis of cellulose and pretreated lignocellulose, Celluclast $1.5 \mathrm{~L}$ (Novozymes), a commercial cellulase preparation produced by T. reesei, was used.

Two types of rice straw pretreated using dilute sulfuric acid and aqueous ammonia were used in this study, which were designated as ACID and ALKALI, respectively. The same pretreated substrates that were previously reported were applied for the synergistic study here [26]. ACID was prepared by using $1 \%(\mathrm{w} / \mathrm{v})$ sulfuric acid at $190{ }^{\circ} \mathrm{C}$ with ramping and holding times for $3 \mathrm{~min}$ and $90 \mathrm{~s}$, respectively, in a microwave digestion system. ALKALI was prepared by soaking in $14 \%(\mathrm{w} / \mathrm{v})$ aqueous ammonia at $69{ }^{\circ} \mathrm{C}$ for $10 \mathrm{~h}$. Then, both pretreated substrates were washed with water and dried at $45^{\circ} \mathrm{C}[27$, 28]. The composition of cellulose, hemicellulose, and lignin in ACID and ALKALI is also described in that study [26]. In brief, the contents of cellulose, hemicellulose, and lignin in untreated rice straw were 36.4 and 18.0, and $18.1 \%(\mathrm{w} / \mathrm{w})$, respectively. In ACID, the contents of cellulose and lignin were 56.9 and $24.8 \%(\mathrm{w} / \mathrm{w})$, respectively, while those of hemicellulosic components such as xylan, galactan, arabinan, and mannan were not determined since most of them were solubilized by acidic treatment. In ALKALI, lignin was substantially removed by alkaline treatment leading to the contents of cellulose, hemicellulose, and lignin being 47.6, and 16.1, and $15.1 \%$ $(\mathrm{w} / \mathrm{w})$, respectively.

\section{Analysis of enzymatic reaction products by MALDI-TOF/ TOF MS}

Enzymatic reactions were performed by incubating 5\% (w/v) of Avicel PH101 (Sigma-Aldrich, St. Louis, MO) with $1 \mathrm{mg}$ of TtAA9E or TaAA9A/g Avicel in $50 \mathrm{mM}$ sodium acetate ( $\mathrm{pH} 5.0)$ at $50{ }^{\circ} \mathrm{C}$ for 4 days. After centrifugation of the reaction mixture, the supernatant containing soluble products was taken for analysis by MALDI-TOF/TOF MS. Prior to the injection into MALDI-TOF/TOF MS, sample was prepared as follows. After purification and desalting of the reaction product, $1 \mu \mathrm{L}$ of the product dissolved in water was spotted onto a stainless steel target plate. Next, sodium saturation was achieved using the solutions including $0.3 \mu \mathrm{L}$ of $0.01 \mathrm{M} \mathrm{NaCl}$ followed by the addition of $0.5 \mu \mathrm{L}$ of $50 \mathrm{mg} /$ $\mathrm{mL} 2,5$-dihydroxybenzoic acid in 50\% (w/v) acetonitrile. Then, the spot solution was subjected to vacuum drying for homogeneous crystallization. An ultrafleXtreme system (Bruker Daltonics, Billerica, MA) with a positive 
ion reflectron mode was used for MALDI-TOF/TOF MS analysis. The detailed analytical methods were presented in a previous study [29]. The calibration of mass spectra was externally carried out using cellooligosaccharides as standards. Processing of raw MS data was performed using a FlexAnalysis software (version 3.3; Bruker Daltonics). Using a signal-to-noise ratio of 3.0, MS peaks were filtered, followed by deconvolution.

\section{Synergistic hydrolysis by cellulase with TtAA9E or TaAA9A}

For the hydrolysis of cellulose, $1 \%(\mathrm{w} / \mathrm{v})$ of Avicel PH-101 was incubated with $0.9 \mathrm{FPU} / \mathrm{g}$ Avicel of Celluclast $1.5 \mathrm{~L}$ in the presence or absence of $1 \mathrm{mg}$ of TtAA9E or TaAA9A/g Avicel in $50 \mathrm{mM}$ sodium acetate buffer (pH 5.0) at $50{ }^{\circ} \mathrm{C}$ for 2 days. For the hydrolysis of pretreated rice straw, $5 \%$ $(\mathrm{w} / \mathrm{v})$ of ACID or ALKALI was incubated with Celluclast $1.5 \mathrm{~L}$ (1.4 and $2.8 \mathrm{FPU} / \mathrm{g}$ total solids) in the presence or absence of $5 \mathrm{mg}$ of TtAA9E or TaAA9A/g cellulose in $50 \mathrm{mM}$ sodium acetate buffer ( $\mathrm{pH}$ 5.0) containing $1 \mathrm{mM}$ $\mathrm{MnSO}_{4}$ at $50{ }^{\circ} \mathrm{C}$ for 8 days. Sodium azide at $0.02 \%(\mathrm{w} / \mathrm{v})$ was added to all the synergistic reactions as an antibiotic or reducing agent. After the reaction, the reaction mixture was boiled at $95{ }^{\circ} \mathrm{C}$ for $5 \mathrm{~min}$ to terminate the reaction, and subjected to centrifugation at $30,000 \times g$ for $5 \mathrm{~min}$. Finally, reducing sugars in the supernatant were quantified by the 3,5-dinitrosalicylic acid (DNS) assay at $540 \mathrm{~nm}$ using D-glucose as the standard. The extent of synergism is represented as the fold increase as follows. Extent of synergism $=$ Reducing sugar yield from the hydrolysis of TtAA9E or TaAA9A with cellulase/Reducing sugar yield from the hydrolysis of cellulase only.

\section{Additional file}

Additional file 1: Figure S1. Expanded mass spectra of reaction products obtained from the incubation of cellulose with TtAA9E or TaAA9A using MALDI-TOF/TOF MS. Avicel (5\%, w/v) was incubated with TtAA9E or TaAA9A (1 mg/g Avicel) in $50 \mathrm{mM}$ sodium acetate ( $\mathrm{pH}$ 5.0) with $10 \mathrm{mM}$ of ascorbic acid at $50^{\circ} \mathrm{C}$ for 4 days. Expanded mass spectra for $\mathbf{a}$ DP5, b DP6, and $\mathbf{c}$ DP7 products obtained from the incubation of cellulose with TtAA9E or TaAA9A. $100 \%$ relative intensity represents $4.62 \times 10^{4}$ a.u. for DP5 and DP6 and $3.00 \times 10^{4}$ a.u. for DP7. Possible products by C1 oxidation are aldonic acid, sodium adduct of aldonic acid, and 1,5 $\delta$-lactone, and those by $\mathrm{C} 4$ oxidation are 4-ketoaldose and gemdiol. Product that contain both aldonic acid and gemdiol, 1,5 $\delta$-lactone and gemdiol, or aldonic acid and 4-ketoaldose is an evidence of double (C1/C4) oxidation. The detailed mass information is shown in the text and Fig. 2.

\section{Abbreviations}

LPMO: Iytic polysaccharide monooxygenase; AA9: auxiliary activity 9; $\mathrm{CDH}$ : cellobiose dehydrogenase; GH61: glycoside hydrolase family 61; MALDI-TOF/TOF MS: matrix-assisted laser desorption/ionization tandem time-of-flight mass spectrometry; DP: degree of polymerization; DNS: 3,5-dinitrosalicylic acid; FPU: filter paper unit.

\section{Authors' contributions}

IJK designed and carried out all the experiments, analyzed the data, and wrote the manuscript. NS, HJA, and JHK performed product analysis. PVH provided
AA9 enzymes, analyzed the data, and wrote the manuscript. KHK conceived the project, analyzed the data, and wrote the manuscript. All authors read and approved the final manuscript.

\section{Author details}

1 Department of Biotechnology, Korea University, Graduate School, Seoul 02841, South Korea. ${ }^{2}$ Graduate School of Analytical Science \& Technology and Asia-Pacific Glycomics Reference Site, Chungnam National University, Daejeon 34134, South Korea. ${ }^{3}$ Department of Food and Nutrition, Chungnam National University, Daejeon 34134, South Korea. ${ }^{4}$ Novozymes, Davis, CA 95618, USA.

\section{Acknowledgements}

This work was performed at the Korea University Food Safety Hall for the Institute of Biomedical Science and Food Safety. The article-processing charge was paid by the Korea University Future Research Grant.

\section{Competing interests}

Novozymes provided the enzymes, and they participated in the analysis of the experimental results. The authors including the author from Novozymes declare that they have no competing interests.

\section{Availability of supporting data}

The supporting data can be found in the Additional file.

\section{Consent for publication}

The authors all agree to publish the paper.

\section{Funding}

We want to acknowledge grant support from C1 Gas Refinery Program through the National Research Foundation of Korea funded by MSIP (2016M3D3A1A01913143).

Received: 15 September 2016 Accepted: 2 February 2017

Published online: 22 February 2017

\section{References}

1. Kim IJ, Lee HJ, Choi I-G, Kim KH. Synergistic proteins for the enhanced enzymatic hydrolysis of cellulose by cellulase. Appl Microbiol Biotechnol. 2014:98:8469-80.

2. Kim ES, Lee HJ, Bang W-G, Choi I-G, Kim KH. Functional characterization of a bacterial expansion from Bacillus subtilis for enhanced enzymatic hydrolysis of cellulose. Biotechnol Bioeng. 2009;102:1342-53.

3. Harris PV, Welner D, MCFarland KC, Re E, Poulsen JCN, Brown K, Salbo R, Ding HS, Vlasenko E, Merino S, Xu F, Cherry J, Larsen S, Lo Leggio L. Stimulation of lignocellulosic biomass hydrolysis by proteins of glycoside hydrolase family 61: structure and function of a large, enigmatic family. Biochemistry. 2010;49:3305-16.

4. Arantes V, Saddler JN. Access to cellulose limits the efficiency of enzymatic hydrolysis: the role of amorphogenesis. Biotechnol Biofuels. 2010:3:4.

5. Beeson WT, Phillips CM, Cate JHD, Marletta MA. Oxidative cleavage of cellulose by fungal copper-dependent polysaccharide monooxygenases. J Am Chem Soc. 2012;134:890-2.

6. Quinlan RJ, Sweeney MD, Lo Leggio L, Otten H, Poulsen JCN, Johansen KS, Krogh K, Jørgensen Cl, Tovborg M, Anthonsen A, Tryfona T, Walter CP, Dupree P, Xu F, Davies GJ, Walton PH. Insights into the oxidative degradation of cellulose by a copper metalloenzyme that exploits biomass components. Proc Natl Acad Sci USA. 2011;108:15079-84.

7. Vaaje-Kolstad G, Westereng B, Horn SJ, Liu ZL, Zhai H, Sørlie M, Eijsink VGH. An oxidative enzyme boosting the enzymatic conversion of recalcitrant polysaccharides. Science. 2010;330:219-22.

8. Phillips CM, Beeson WT, Cate JH, Marletta MA. Cellobiose dehydrogenase and a copper-dependent polysaccharide monooxygenase potentiate cellulose degradation by Neurospora crassa. ACS Chem Biol. 2011;6:1399-406

9. Kim IJ, Nam KH, Yun EJ, Kim S, Youn HJ, Lee HJ, Choi I-G, Kim KH. Optimization of synergism of a recombinant auxiliary activity 9 from Chaetomium 
globosum with cellulase in cellulose hydrolysis. Appl Microbiol Biotechnol. 2015;99:8537-47.

10. Dimarogona M, Topakas E, Olsson L, Christakopoulos P. Lignin boosts the cellulase performance of a GH-61 enzyme from Sporotrichum thermophile. Bioresour Technol. 2012;110:480-7.

11. Hu J, Arantes V, Pribowo A, Gourlay K, Saddler JN. Substrate factors that influence the synergistic interaction of AA9 and cellulases during the enzymatic hydrolysis of biomass. Energy Environ Sci. 2014;7:2308-15.

12. Rodríguez-Zúñiga UF, Cannella D, Giordano R, Giordano RD, JørgensenH, Felby C. Lignocellulose pretreatment technologies affect the level of enzymatic cellulose oxidation by LPMO. Green Chem. 2015:17:2896-903.

13. Kracher D, Scheiblbrandner S, Felice AKG, BresImayr E, Preims M, Ludwicka K, Haltrich D, Eijsink VGH, Ludwig R. Extracellular electron transfer systems fuel cellulose oxidative degradation. Science. 2016;352:1098.

14. Eibinger M, Ganner T, Bubner P, Rošker S, Kracher D, Haltrich D, Ludwig R, Plank H, Nidetzky B. Cellulose surface degradation by a lytic polysaccharide monooxygenase and its effect on cellulase hydrolytic efficiency. J Biol Chem. 2014;289:35929-38.

15. Vermaas JV, Crowley MF, Beckham GT, Payne CM. Effects of lytic polysaccharide monooxygenase oxidation on cellulose structure and binding of oxidized cellulose oligomers to cellulases. J Phys Chem B. 2015:119:6129-43.

16. Levasseur A, Drula E, Lombard V, Coutinho P, Henrissat B. Expansion of the enzymatic repertoire of the CAZy database to integrate auxiliary redox enzymes. Biotechnol Biofuels. 2013;6:41.

17. Busk P, Lange L. Classification of fungal and bacterial lytic polysaccharide monooxygenases. BMC Genom. 2015;16:368.

18. Vu V, Beeson WT, Phillips CM, Cate JHD, Marletta MA. Determinants of regioselective hydroxylation in the fungal polysaccharide monooxygenases. J Am Chem Soc. 2014;136:562-5.

19. Forsberg Z, Mackenzie AK, Sørlie M, Røhr ÅK, Helland R, Arvai AS, Vaaje-Kolstad G, Eijsink VGH. Structural and functional characterization of a conserved pair of bacterial cellulose-oxidizing lytic polysaccharide monooxygenases. Proc Natl Acad Sci USA. 2014;111:8446-51.

20. Langston JA, Shaghasi T, Abbate E, Xu F, Vlasenko E, Sweeney MD. Oxidoreductive cellulose depolymerization by the enzymes cellobiose dehydrogenase and glycoside hydrolase 61. Appl Environ Microbiol. 2011;77:7007-15.
21. Westereng B, Ishida T, Vaaje-Kolstad G, Wu M, Eijsink VGH, Igarashi K, Samejima M, Ståhlberg J, Horn SJ, Sandgren M. The putative endoglucanase PCGH61D from Phanerochaete chrysosporium is a metal-dependent oxidative enzyme that cleaves cellulose. PLOS ONE. 2011;6:e27807.

22. Banerjee G, Car S, Scott-Craig JS, Borrusch MS, Walton JD. Rapid optimization of enzyme mixtures for deconstruction of diverse pretreatment/ biomass feedstock combinations. Biotechnol Biofuels. 2010;3:22.

23. Billard H, Faraj A, Lopes Ferreira N, Menir S, Heiss-Blanquet S. Optimization of a synthetic mixture composed of major Trichoderma reesei enzymes for the hydrolysis of steam-exploded wheat straw. Biotechnol Biofuels. 2012;5:9.

24. Gao D, Chundawat SPS, Krishnan C, Balan V, Dale BE. Mixture optimization of six core glycosyl hydrolases for maximizing saccharification of ammonia fiber expansion (AFEX) pretreated corn stover. Bioresour Technol. 2010;101:2770-81.

25. Müller G, Várnai A, Johansen KS, Eijsink VGH, Horn SJ. Harnessing the potential of LPMO-containing cellulase cocktails poses new demands on processing conditions. Biotechnol Biofuels. 2015;8:187.

26. Kim IJ, Jung JY, Lee HJ, Park HS, Jung YH, Park K, Kim KH. Customized optimization of cellulase mixtures for differently pretreated rice straw. Bioprocess Biosyst Eng. 2015;38:929-37.

27. Kim KH, Tucker MP, Nguyen QA. Effects of pressing lignocellulosic biomass on sugar yield in two-stage dilute-acid hydrolysis process. Biotechnol Prog. 2002;18:489-94.

28. Ko JK, Bak JS, Jung MW, Lee HJ, Choi I-G, Kim TH, Kim KH. Ethanol production from rice straw using optimized aqueous-ammonia soaking pretreatment and simultaneous saccharification and fermentation processes. Bioresour Technol. 2009;100:4374-80.

29. Lee CH, Kim HT, Yun EJ, Lee AR, Kim SR, Kim J-H, Choi I-G, Kim KH. A novel agarolytic $\beta$-galactosidase acts on agarooligosaccharides for complete hydrolysis of agarose into monomers. Appl Environ Microbiol. 2014;80:5965-73

\section{Submit your next manuscript to BioMed Central and we will help you at every step:}

- We accept pre-submission inquiries

- Our selector tool helps you to find the most relevant journal

- We provide round the clock customer support

- Convenient online submission

- Thorough peer review

- Inclusion in PubMed and all major indexing services

- Maximum visibility for your research

Submit your manuscript at www.biomedcentral.com/submit
() Biomed Central 